\title{
On Compact and Noncompact Structures for the Improved Boussinesq Water Equations
}

\author{
Jun Yin ${ }^{1,2}$ and Hongyi Chen ${ }^{3}$ \\ ${ }^{1}$ Institute for Fiscal Science Research, Ministry of Finance of China, Beijing 100142, China \\ ${ }^{2}$ Panzhihua Municipal Finance Bureau of China, Panzhihua, Sichuan 617000, China \\ ${ }^{3}$ Business School, Imperial College London, South Kensington Campus, Exhibition Road, London SW7 2AZ, UK
}

Correspondence should be addressed to Jun Yin; yinjun520777@sohu.com

Received 3 November 2013; Accepted 15 November 2013

Academic Editor: Shaoyong Lai

Copyright (C) 2013 J. Yin and H. Chen. This is an open access article distributed under the Creative Commons Attribution License, which permits unrestricted use, distribution, and reproduction in any medium, provided the original work is properly cited.

\begin{abstract}
The nonlinear variants of the generalized Boussinesq water equations with positive and negative exponents are studied in this paper. The analytic expressions of the compactons, solitons, solitary patterns, and periodic solutions for the equations are obtained by using a technique based on the reduction of order of differential equations. It is shown that the nonlinear variants, or nonlinear variants together with the wave numbers, directly lead to the qualitative change in the physical structures of the solutions.
\end{abstract}

\section{Introduction}

One of the classical Boussinesq water equations can be written by the form

$$
u_{t t}=-\alpha u_{x x x x}+u_{x x}+\beta\left(u^{2}\right)_{x x}
$$

where $u(x, t)$ is the elevation of the free surface of fluid, the subscripts denote partial derivatives, and the constant coefficients $\alpha$ and $\beta$ depend on the depth of fluid and the characteristic speed of the long waves. It is well known that Boussinesq equation (1) was originally introduced as a model for one-dimensional weakly nonlinear dispersive waves in shallow water and was subsequently applied to explain problems in the percolation of water in porous subsurface strata. It also crops up in the analysis of many other physical processes.

Various generalizations of the classical Boussinesq water equation have been proposed and studied to probe the dynamic properties of their solutions. The generalized Boussinesq's equations have been successfully applied in coastal engineering for simulating wave propagation from the deep sea to a shallow-water region and in naval architecture for computing ship waves in shallow water (See $[1,2])$. Kaya [3] obtained the exact and numerical solitary-wave solutions for a generalized modified Boussinesq equation.
Elgarayhi and Elhanbaly [1] discussed and analyzed new exact traveling wave solutions for the two-dimensional KdVBurgers and Boussinesq equations. Lai and $\mathrm{Wu}[4]$ and Lai et al. [5] proposed an approach for constructing asymptotic solution of the Boussinesq equations. Rosenau [6] proved that the vibration of the anharmonic mass-spring chain leads to a new Boussinesq equation admitting compactons and compact breathers. It was formally shown that the collision of two compactons results in the creation of low-amplitude compacton and anticompacton pairs. One can find that many meaningful achievements that outline the structure of compactons and the collision of two compactons were reported in [6-8]. For more details about the compactons and soliton solutions for nonlinear evolution equations, the reader is referred to $[4,5,9-11]$ in which many analytical and numerical methods such as the pseudospectral methods, the Galerkin method, the finite differences method, the sinecosine ansatz, and the tanh method have been presented.

The following $(2+1)$-dimensional Boussinesq water system

$$
u_{t t}-u_{x x}-u_{y y}-\left(u^{2}\right)_{x x}-u_{x x x x}=0
$$

was studied in [11]. The author discussed the stability of solitary wave solution of (2). It is found that pulse-like 
solutions to the Boussinesq water equation are stable to linear perturbations.

Wazwaz [12] studied the variants of the following improved Boussinesq water equations.

(I) Variant of the $(1+1)$-dimensional improved Boussinesq equation with positive exponents is given by

$$
u_{t t}-u_{x x}-a\left(u^{2 n}\right)_{x x}-b\left[u^{n}\left(u^{n}\right)_{x x}\right]_{t t}=0, \quad n>1,
$$

and with negative exponents is given by

$$
u_{t t}-u_{x x}-a\left(u^{-2 n}\right)_{x x}-b\left[u^{-n}\left(u^{-n}\right)_{x x}\right]_{t t}=0, \quad n>1,
$$

where $a \neq 0$ and $b \neq 0$ are constants.

(II) Variant of the $(2+1)$ dimensional improved Boussinesq equation with positive exponents (some typing's errors occurred for (5) and (6) in [12]. This can be found through the reading of the whole paper) is given by

$$
\begin{aligned}
u_{t t} & -u_{x x}-u_{y y}-a_{1}\left(u^{2 n}\right)_{x x}-a_{2}\left(u^{2 n}\right)_{y y} \\
& -b_{1}\left[u^{n}\left(u^{n}\right)_{x x}\right]_{t t} \\
& -b_{2}\left[u^{n}\left(u^{n}\right)_{y y}\right]_{t t}=0, \quad n>1,
\end{aligned}
$$

and with negative exponents is in the form

$$
\begin{aligned}
u_{t t} & -u_{x x}-u_{y y}-a_{1}\left(u^{-2 n}\right)_{x x}-a_{2}\left(u^{-2 n}\right)_{y y} \\
& -b_{1}\left[u^{-n}\left(u^{-n}\right)_{x x}\right]_{t t} \\
& -b_{2}\left[u^{-n}\left(u^{-n}\right)_{y y}\right]_{t t}=0, \quad n>1,
\end{aligned}
$$

where $a_{1}+a_{2} \neq 0$ and $b_{1}+b_{2} \neq 0$.

Wazwaz [12] assumed that the solutions of (3), (4), (5), and (6) take the forms in terms of sine or cosine; namely,

$$
u(x, t)=\left\{\lambda \cos ^{\beta}\left(\mu \xi_{1}\right)\right\}, \quad\left|\xi_{1}\right| \leq \frac{\pi}{2 \mu},
$$

or the ansatz

$$
u(x, t)=\left\{\lambda \sin ^{\beta}\left(\mu \xi_{1}\right)\right\}, \quad\left|\xi_{1}\right| \leq \frac{\pi}{\mu},
$$

where $\xi_{1}=x-c t$ or $\xi_{1}=x+y-c t$ and $\mu$ and $c$ are the wave number and the wave speed, respectively. Using the balancing rule, Wazwaz [12] calculated and found out the concrete values of $\beta, \mu, \lambda$.

In this paper, by using a mathematical technique different from those in $[9,12]$, we derive analytical expressions of the travelling solutions with wave variable $\xi=\mu(x-c t)$ for (3) and (4) or $\xi=\mu x+\eta y-c t$ for (5) and (6) where $\mu, \eta$, and $c$ are arbitrary nonzero constants. The technique used in this paper is proved very useful in constructing the exact travelling wave solutions for (3)-(6). It is shown that the nonlinear variants, or nonlinear variants together with the wave numbers, directly determine the physical structures of the solutions such as compactons, solitons, solitary patterns, and periodic solutions. Moreover, the results presented in this paper include those carried out in Wazwaz [12].

\section{Variant of the $(1+1)$-Dimensional Improved Boussinesq Equation with Positive Exponents}

Firstly, we consider solutions of the following equation:

$$
\left(\frac{d W}{d z}\right)^{2}=a_{0}-b_{0} W^{2},
$$

where $a_{0} \neq 0$ and $b_{0} \neq 0$ are constants. When $b_{0}>0,(9)$ admits two solutions:

$$
\begin{aligned}
& W_{1}= \pm \sqrt{\frac{a_{0}}{b_{0}}} \sin \left[\sqrt{b_{0}}(z+A)\right], \\
& W_{2}= \pm \sqrt{\frac{a_{0}}{b_{0}}} \cos \left[\sqrt{b_{0}}(z+A)\right],
\end{aligned}
$$

where $A$ is an arbitrary constant.

When $b_{0}<0$, noticing that $\cosh ^{2} z-\sinh ^{2} z=1$, we derive that (9) has two solutions of the form

$$
\begin{aligned}
& W_{3}= \pm \sqrt{-\frac{a_{0}}{b_{0}}} \sinh \left[\sqrt{-b_{0}}(z+A)\right], \\
& W_{4}= \pm i \sqrt{-\frac{a_{0}}{b_{0}}} \cosh \left[\sqrt{-b_{0}}(z+A)\right],
\end{aligned}
$$

where $i=\sqrt{-1}$.

Now, we write out the following formulas which will be used in this work:

$$
\begin{aligned}
\sinh x & =\frac{1}{i} \sin i x, & \sin x & =\frac{1}{i} \sinh i x, \\
\cosh x & =\cos i x, & \cos x & =\cosh i x .
\end{aligned}
$$

Remark 1. Here we point out that we will solve (9) in the complex value district. By the theory of solution structure for the first-order ordinary differential equation, we know that the formulas (10) and (11) include all the analytical solutions of (9). In the following discussions, we let the phase number $A=0$ in formulas (10), (11) and the forthcoming analytical expressions of travelling wave solutions.

In this section, we seek formal travelling wave solutions for (3); namely,

$$
u(x, t)=u(\xi)
$$

where the wave variable $\xi$ takes the form $\xi=\mu(x-c t)$ with constants $\mu \neq 0$ and $c \neq 0$. ODE:

The wave variable $\xi=\mu(x-c t)$ turns (3) into the following

$$
\left(c^{2}-1\right) u_{\xi \xi}-a\left(u^{2 n}\right)_{\xi \xi}-b c^{2} \mu^{2}\left[u^{n}\left(u^{n}\right)_{\xi \xi}\right]_{\xi \xi}=0 .
$$

Integrating (14) twice and setting the constants of integration to be zero, we have

$$
\left(c^{2}-1\right) u-a u^{2 n}-b c^{2} \mu^{2} u^{n}\left(u^{n}\right)_{\xi \xi}=0 .
$$



holds.

Since $c^{2}$ appears in (15), we can assume that $c>0$ always

Case 1. When $c^{2}=1$, if $a / b<0$, the solution of (15) takes the form

$$
u^{n}=k_{1} \exp \left(\sqrt{-\frac{a}{b}}(x-c t)\right)+k_{2} \exp \left(-\sqrt{-\frac{a}{b}}(x-c t)\right)
$$

where $k_{1}$ and $k_{2}$ are arbitrary constants.

If $a / b>0$, we solve (15) by

$$
u^{n}=k_{3} \sin \left(\sqrt{\frac{a}{b}}(x-c t)\right)+k_{4} \cos \left(\sqrt{\frac{a}{b}}(x-c t)\right) .
$$

Here $k_{3}$ and $k_{4}$ are arbitrary constants.

Case 2. When $c^{2} \neq 1$, letting $d u^{n} / d \xi=Z$, we have $d^{2} u^{n} / d \xi^{2}=$ $Z\left(d Z / d u^{n}\right)$ and

$$
\left(c^{2}-1\right) u-a u^{2 n}-b c^{2} \mu^{2} u^{n} Z \frac{d Z}{d u^{n}}=0 .
$$

Using $d u^{n}=n u^{n-1} d u$, we have

$$
\left(c^{2}-1\right)-a u^{2 n-1}-\frac{b c^{2} \mu^{2}}{n} Z \frac{d Z}{d u}=0 .
$$

Further calculation of (19) gives rise to

$$
\left(\frac{d u^{(2 n-1) / 2}}{d \xi}\right)^{2}=\frac{(2 n-1)^{2}}{b c^{2} \mu^{2}(2 n)^{2}}\left[2 n\left(c^{2}-1\right)-a\left(u^{(2 n-1) / 2}\right)^{2}\right] .
$$

If $a / b>0$, it follows from (10) and (20) that

$$
\begin{aligned}
& u^{(2 n-1) / 2} \\
& \quad= \pm \sqrt{\frac{2 n\left(c^{2}-1\right)}{a}} \sin \left\{\frac{(2 n-1)}{2 n c \mu} \sqrt{\frac{a}{b}}[\mu(x-c t)+A]\right\},
\end{aligned}
$$

or

$$
\begin{aligned}
& u^{(2 n-1) / 2} \\
& \quad= \pm \sqrt{\frac{2 n\left(c^{2}-1\right)}{a}} \cos \left\{\frac{(2 n-1)}{2 n c \mu} \sqrt{\frac{a}{b}}[\mu(x-c t)+A]\right\},
\end{aligned}
$$

where $A$ is a free constant. Choosing the phase number $A=0$ in formulas (21) and (22) and limiting the domain of $\xi$, we have the following compacton solutions:

$$
\begin{array}{r}
u=\left\{\frac{2 n\left(c^{2}-1\right)}{a} \sin ^{2}\left[\frac{(2 n-1)}{2 n c} \sqrt{\frac{a}{b}}(x-c t)\right]\right\}^{1 /(2 n-1)}, \\
|(x-c t)|<\frac{2 n c \pi}{(2 n-1) \sqrt{a / b}},
\end{array}
$$

$u=0, \quad$ otherwise,

$$
\begin{gathered}
u=\left\{\frac{2 n\left(c^{2}-1\right)}{a} \cos ^{2}\left[\frac{(2 n-1)}{2 n c} \sqrt{\frac{a}{b}}(x-c t)\right]\right\}^{1 /(2 n-1)}, \\
|(x-c t)|<\frac{n c \pi}{(2 n-1) \sqrt{a / b}},
\end{gathered}
$$

$u=0, \quad$ otherwise

However, for $a / b<0$, from (11) and (20), we obtain the solitary pattern solutions:

$$
\begin{aligned}
& u=\left\{-\frac{2 n\left(c^{2}-1\right)}{a} \sinh ^{2}\left[\frac{(2 n-1)}{2 n c} \sqrt{-\frac{a}{b}}(x-c t)\right]\right\}^{1 /(2 n-1)}, \\
& u=\left\{\frac{2 n\left(c^{2}-1\right)}{a} \cosh ^{2}\left[\frac{(2 n-1)}{2 n c} \sqrt{-\frac{a}{b}}(x-c t)\right]\right\}^{1 /(2 n-1)} .
\end{aligned}
$$

Remark 2. From the formulas (21)-(22), we conclude that the compactons and solitary pattern solutions are independent of wave number $\mu$ when we choose the phase number equals zero. These results extend those obtained by Wazwaz [12] in which the wave number $\mu$ was chosen as a special constant.

\section{Variant of the $(1+1)$-Dimensional Improved Boussinesq Equation with Negative Exponents}

Now, we consider the (1+1)-dimensional improved Boussinesq equation with negative exponents; namely,

$$
\begin{aligned}
u_{t t} & -u_{x x}-a\left(u^{-2 n}\right)_{x x} \\
& -b\left[u^{-n}\left(u^{-n}\right)_{x x}\right]_{t t}=0, \quad n>1, a \neq 0, \quad b \neq 0 .
\end{aligned}
$$


Since (3) and (26) are symmetric about $n$ and $(-n)$, we can readily obtain the following periodic solutions for (26):

$$
\begin{gathered}
u=\left\{-\frac{a}{2 n\left(c^{2}-1\right)} \csc ^{2}\left[\frac{(2 n+1)}{2 n c} \sqrt{\frac{a}{b}}(x-c t)\right]\right\}^{1 /(2 n+1)}, \\
\frac{a}{b}>0, c \neq \pm 1, \\
u=\left\{-\frac{a}{2 n\left(c^{2}-1\right)} \sec ^{2}\left[\frac{(2 n+1)}{2 n c} \sqrt{\left.\left.\frac{a}{b}(x-c t)\right]\right\}^{1 /(2 n+1)},}\right.\right. \\
\frac{a}{b}>0, c \neq \pm 1 .
\end{gathered}
$$

For the case where $a / b<0,(26)$ has solitons of the form

$$
\begin{gathered}
u=\left\{\frac{a}{2 n\left(c^{2}-1\right)} \operatorname{csch}^{2}\left[\frac{(2 n+1)}{2 n c} \sqrt{-\frac{a}{b}}(x-c t)\right]\right\}^{1 /(2 n+1)}, \\
c \neq \pm 1, \\
u=\left\{-\frac{a}{2 n\left(c^{2}-1\right)} \operatorname{sech}^{2}\left[\frac{(2 n+1)}{2 n c} \sqrt{-\frac{a}{b}}(x-c t)\right]\right\}^{1 /(2 n+1)}, \\
c \neq \pm 1 .
\end{gathered}
$$

\section{Variant of the (2+1)-Dimensional Improved Boussinesq Equation with Positive Exponents}

In this section, we consider the solution of (5). The use of wave variable $\xi=\mu x+\eta y-c t$ carries the equation into the following ordinary differential equation (ODE):

$$
\begin{gathered}
\left(c^{2}-\mu^{2}-\eta^{2}\right) u_{\xi \xi}-\left(a_{1} \mu^{2}+a_{2} \eta^{2}\right)\left(u^{2 n}\right)_{\xi \xi} \\
-\left(b_{1} \mu^{2}+b_{2} \eta^{2}\right) c^{2}\left(u^{n}\left(u^{n}\right)_{\xi \xi}\right)_{\xi \xi}=0 .
\end{gathered}
$$

Integrating (29) twice and setting the constants of integration to be zero, we get

$$
\begin{gathered}
\left(c^{2}-\mu^{2}-\eta^{2}\right) u-\left(a_{1} \mu^{2}+a_{2} \eta^{2}\right) u^{2 n} \\
-\left(b_{1} \mu^{2}+b_{2} \eta^{2}\right) c^{2} u^{n}\left(u^{n}\right)_{\xi \xi}=0 .
\end{gathered}
$$

Using the transformation $d u^{n} / d \xi=Z$, we have $d^{2} u^{n} / d \xi^{2}=$ $Z\left(d Z / d u^{n}\right)$ and

$$
\begin{gathered}
\left(c^{2}-\mu^{2}-\eta^{2}\right) u-\left(a_{1} \mu^{2}+a_{2} \eta^{2}\right)\left(u^{2 n}\right) \\
-\left(b_{1} \mu^{2}+b_{2} \eta^{2}\right) c^{2} u^{n} Z \frac{d Z}{d u^{n}}=0 .
\end{gathered}
$$

Furthermore,

$$
\begin{gathered}
\left(c^{2}-\mu^{2}-\eta^{2}\right)-\left(a_{1} \mu^{2}+a_{2} \eta^{2}\right)\left(u^{2 n-1}\right) \\
-\left(b_{1} \mu^{2}+b_{2} \eta^{2}\right) c^{2} Z \frac{d Z}{n d u}=0 .
\end{gathered}
$$

In the following discussions, we assume that $\left(c^{2}-\mu^{2}-\right.$ $\left.\eta^{2}\right) \neq 0, a_{1} \mu^{2}+a_{2} \eta^{2} \neq 0$, and $b_{1} \mu^{2}+b_{2} \eta^{2} \neq 0$.

Integrating (32) yields

$$
\begin{aligned}
& \frac{\left(b_{1} \mu^{2}+b_{2} \eta^{2}\right) c^{2}}{2 n} Z^{2} \\
& \quad=\left(c^{2}-\mu^{2}-\eta^{2}\right) u-\left(a_{1} \mu^{2}+a_{2} \eta^{2}\right) \frac{1}{2 n}\left(u^{2 n}\right) .
\end{aligned}
$$

It follows from (33) that

$$
\frac{\left(b_{1} \mu^{2}+b_{2} \eta^{2}\right) c^{2}}{2 n}\left(\frac{2 n d u^{(2 n-1) / 2}}{(2 n-1) d \xi}\right)^{2}
$$

$$
=\left(c^{2}-\mu^{2}-\eta^{2}\right)-\left(a_{1} \mu^{2}+a_{2} \eta^{2}\right) \frac{1}{2 n}\left(u^{2 n-1}\right) \text {. }
$$

Setting $W=u^{(2 n-1) / 2}$, we have

$$
\begin{aligned}
\left(\frac{d W}{d \xi}\right)^{2}= & \frac{(2 n-1)^{2}}{2 n c^{2}\left(b_{1} \mu^{2}+b_{2} \eta^{2}\right)} \\
& \times\left[\left(c^{2}-\mu^{2}-\eta^{2}\right)-\left(a_{1} \mu^{2}+a_{2} \eta^{2}\right) \frac{1}{2 n} W^{2}\right] .
\end{aligned}
$$

(1) If $\left(a_{1} \mu^{2}+a_{2} \eta^{2}\right) /\left(b_{1} \mu^{2}+b_{2} \eta^{2}\right)>0$ and $n>1 / 2$, it follows from (10) and (35) that

$$
\begin{aligned}
& W=\sqrt{\frac{2 n\left(c^{2}-\mu^{2}-\eta^{2}\right)}{a_{1} \mu^{2}+a_{2} \eta^{2}}} \sin \left[\frac{2 n-1}{2 n c} \sqrt{\frac{a_{1} \mu^{2}+a_{2} \eta^{2}}{b_{1} \mu^{2}+b_{2} \eta^{2}}}(\xi+A)\right], \\
& W=\sqrt{\frac{2 n\left(c^{2}-\mu^{2}-\eta^{2}\right)}{a_{1} \mu^{2}+a_{2} \eta^{2}}} \cos \left[\frac{2 n-1}{2 n c} \sqrt{\frac{a_{1} \mu^{2}+a_{2} \eta^{2}}{b_{1} \mu^{2}+b_{2} \eta^{2}}}(\xi+A)\right] .
\end{aligned}
$$

Letting $A=0$ in formulas (36), we have the following compacton solutions:

$$
\begin{aligned}
u=\left\{\frac{2 n\left(c^{2}-\mu^{2}-\eta^{2}\right)}{a_{1} \mu^{2}+a_{2} \eta^{2}}\right. \\
\left.\quad \times \sin ^{2}\left[\frac{2 n-1}{2 n c} \sqrt{\frac{a_{1} \mu^{2}+a_{2} \eta^{2}}{b_{1} \mu^{2}+b_{2} \eta^{2}}}(\mu x+\eta y-c t)\right]\right\}^{1 /(2 n-1)}, \\
|(\mu x+\eta y-c t)|<\frac{2 n c \pi}{(2 n-1)} \sqrt{\frac{b_{1} \mu^{2}+b_{2} \eta^{2}}{a_{1} \mu^{2}+a_{2} \eta^{2}}},
\end{aligned}
$$

$u=0, \quad$ otherwise, 


$$
\begin{aligned}
u=\left\{\frac{2 n\left(c^{2}-\mu^{2}-\eta^{2}\right)}{a_{1} \mu^{2}+a_{2} \eta^{2}}\right. \\
\left.\quad \times \cos ^{2}\left[\frac{2 n-1}{2 n c} \sqrt{\frac{a_{1} \mu^{2}+a_{2} \eta^{2}}{b_{1} \mu^{2}+b_{2} \eta^{2}}}(\mu x+\eta y-c t)\right]\right\}^{1 /(2 n-1)}, \\
|(\mu x+\eta y-c t)|<\frac{n c \pi}{(2 n-1)} \sqrt{\frac{b_{1} \mu^{2}+b_{2} \eta^{2}}{a_{1} \mu^{2}+a_{2} \eta^{2}}},
\end{aligned}
$$

$u=0, \quad$ otherwise.

(2) For $a_{1} \mu^{2}+a_{2} \eta^{2} / b_{1} \mu^{2}+b_{2} \eta^{2}<0$ and $n>1 / 2$, we obtain the solitary pattern solutions

$$
\begin{gathered}
u=\left\{-\frac{2 n\left(c^{2}-\mu^{2}-\eta^{2}\right)}{a_{1} \mu^{2}+a_{2} \eta^{2}}\right. \\
\times \sinh ^{2}\left[\frac{2 n-1}{2 n c} \sqrt{-\frac{a_{1} \mu^{2}+a_{2} \eta^{2}}{b_{1} \mu^{2}+b_{2} \eta^{2}}}\right. \\
u=\left\{\begin{array}{c}
\frac{2 n\left(c^{2}-\mu^{2}-\eta^{2}\right)}{a_{1} \mu^{2}+a_{2} \eta^{2}} \\
\times \cosh ^{2}\left[\frac{2 n-1}{2 n c} \sqrt{-\frac{a_{1} \mu^{2}+a_{2} \eta^{2}}{b_{1} \mu^{2}+b_{2} \eta^{2}}}\right. \\
\times(\mu x+\eta y-c t)]\}^{1 /(2 n-1)}
\end{array}\right.
\end{gathered}
$$

Letting $\mu=\eta$ and $c=\mu c_{1}$ in formulas (37) to (40), we obtain the analytical expressions of compactons and solitary pattern solutions for (5) which was established in Wazwaz's paper [12].

\section{Variant of the $(2+1)$-Dimensional Improved Boussinesq Equation with Negative Exponents}

We firstly assume that $\left(c^{2}-\mu^{2}-\eta^{2}\right) \neq 0, a_{1} \mu^{2}+a_{2} \eta^{2} \neq 0$, and $b_{1} \mu^{2}+b_{2} \eta^{2} \neq 0$. Taking the symmetric property of $(-n)$ and $n$ into account in (5) and (6), we find the periodic solutions in the case where $\left(a_{1} \mu^{2}+a_{2} \eta^{2}\right) /\left(b_{1} \mu^{2}+b_{2} \eta^{2}\right)>0$ for (6) as follows:

$$
\begin{aligned}
u= & \left\{-\frac{a_{1} \mu^{2}+a_{2} \eta^{2}}{2 n\left(c^{2}-\mu^{2}-\eta^{2}\right)}\right. \\
& \left.\times \csc ^{2}\left[\frac{2 n+1}{2 n c} \sqrt{\frac{a_{1} \mu^{2}+a_{2} \eta^{2}}{b_{1} \mu^{2}+b_{2} \eta^{2}}}(\mu x+\eta y-c t)\right]\right\}^{1 /(2 n+1)}, \\
u= & \left\{-\frac{a_{1} \mu^{2}+a_{2} \eta^{2}}{2 n\left(c^{2}-\mu^{2}-\eta^{2}\right)},\right. \\
& \left.\times \cos ^{2}\left[\frac{2 n+1}{2 n c} \sqrt{\frac{a_{1} \mu^{2}+a_{2} \eta^{2}}{b_{1} \mu^{2}+b_{2} \eta^{2}}}(\mu x+\eta y-c t)\right]\right\}^{1 /(2 n+1)},
\end{aligned}
$$

where $n>-1 / 2$.

If $\left(a_{1} \mu^{2}+a_{2} \eta^{2}\right) /\left(b_{1} \mu^{2}+b_{2} \eta^{2}\right)<0$, we obtain the solitons

$$
\begin{aligned}
u= & \left\{\frac{a_{1} \mu^{2}+a_{2} \eta^{2}}{2 n\left(c^{2}-\mu^{2}-\eta^{2}\right)}\right. \\
& \left.\times \operatorname{csch}^{2}\left[\frac{2 n+1}{2 n c} \sqrt{-\frac{a_{1} \mu^{2}+a_{2} \eta^{2}}{b_{1} \mu^{2}+b_{2} \eta^{2}}}(\mu x+\eta y-c t)\right]\right\}^{1 /(2 n+1)}, \\
u= & \left\{-\frac{a_{1} \mu^{2}+a_{2} \eta^{2}}{2 n\left(c^{2}-\mu^{2}-\eta^{2}\right)},\right. \\
& \left.\times \operatorname{sech}^{2}\left[\frac{2 n+1}{2 n c} \sqrt{-\frac{a_{1} \mu^{2}+a_{2} \eta^{2}}{b_{1} \mu^{2}+b_{2} \eta^{2}}}(\mu x+\eta y-c t)\right]\right\}^{1 /(2 n+1)},
\end{aligned}
$$

where $n>-1 / 2$.

Also, choosing $\mu=\eta$ and $c=\mu c_{1}$ in (42) produces the analytical expressions of periodic and soliton solutions for (6) which was obtained in Wazwaz [12].

Remark 3. From the formulae (37) to (46), we can conclude that the exponent $n$, the wave numbers $\mu$ and $\eta$, and the coefficients $a_{1}, a_{2}, b_{1}$, and $b_{2}$ appearing in Boussinesq equation (5) or (6) are the main factors which determine the change of physical structures of solutions. More precisely, exponent $n$ and number $\left(a_{1} \mu^{2}+a_{2} \eta^{2}\right) /\left(b_{1} \mu^{2}+b_{2} \eta^{2}\right)$, positive or negative, directly result in the qualitative changes in the physical structures of solutions.

\section{Conclusion}

In this paper, we solve the generalized Boussinesq water equations with positive or negative exponents. The analytical expressions of the travelling wave solutions for the nonlinear equations presented by (3)-(6) are established.

For the $(1+1)$-dimensional Boussinesq equations, two outcomes are achieved. One is that the wave number $\mu$ in the analytically expressions of the travelling waves solutions keeps as an arbitrary nonzero constant when the corresponding phase equals zero. In other words, the travelling wave 
solutions are independent of the wave number $\mu$ if the phase of the solutions is zero. The other is that the exponent $n$ together with the ratio $a / b$, positive or negative, directly determines the qualitative changes in the physical structures of solutions.

For the (2+1)-dimensional generalized Boussinesq equations with positive or negative exponents, we obtain analytical expressions for the compactons, solitons, solitary patterns, and periodic solutions. It is shown that the exponent $n$, the wave numbers $\mu$ and $\eta$, and the coefficients $a_{1}, a_{2}, b_{1}$, and $b_{2}$ appearing in Boussinesq equation (5) and (6) are the main factors which determine the changes of physical structures of solutions. More precisely, the exponent $n$ and the factor $\left(a_{1} \mu^{2}+a_{2} \eta^{2}\right) /\left(b_{1} \mu^{2}+b_{2} \eta^{2}\right)$, positive or negative, directly lead to the qualitative changes in the physical structures of the solutions.

\section{References}

[1] A. Elgarayhi and A. Elhanbaly, "New exact traveling wave solutions for the two-dimensional KdV-Burgers and Boussinesq equations," Physics Letters A, vol. 343, no. 1-3, pp. 85-89, 2005.

[2] O. Nwogu, "Alternative form of Boussinesq equations for nearshore wave propagation," Journal of Waterway, Port, Coastal \& Ocean Engineering, vol. 119, no. 6, pp. 618-638, 1993.

[3] D. Kaya, "The exact and numerical solitary-wave solutions for generalized modified Boussinesq equation," Physics Letters A, vol. 348, no. 3-6, pp. 244-250, 2006.

[4] S. Y. Lai and Y. H. Wu, "The asymptotic solution of the Cauchy problem for a generalized Boussinesq equation," Discrete and Continuous Dynamical Systems B, vol. 3, no. 3, pp. 401-408, 2003.

[5] S. Y. Lai, Y. H. Wu, and X. Yang, "The global solution of an initial boundary value problem for the damped Boussinesq equation," Communications on Pure and Applied Analysis, vol. 3, no. 2, pp. 319-328, 2004.

[6] P. Rosenau, "Nonlinear dispersion and compact structures," Physical Review Letters, vol. 70, no. 5, pp. 564-567, 1993.

[7] P. J. Olver and P. Rosenau, "Tri-Hamiltonian duality between solitons and solitary-wave solutions having compact support," Physical Review E, vol. 53, no. 2, pp. 1900-1906, 1996.

[8] P. Rosenau, "On a class of nonlinear dispersive-dissipative interactions," Physica D, vol. 123, no. 1-4, pp. 525-546, 1998.

[9] A.-M. Wazwaz, "Existence and construction of compacton solutions," Chaos, Solitons and Fractals, vol. 19, no. 3, pp. 463470, 2004.

[10] M. S. Ismail and T. R. Taha, "A numerical study of compactons," Mathematics and Computers in Simulation, vol. 47, no. 6, pp. 519-530, 1998.

[11] Y. Zheng and S. Lai, "Peakons, solitary patterns and periodic solutions for generalized Camassa-Holm equations," Physics Letters A, vol. 372, no. 23, pp. 4141-4143, 2008.

[12] A.-M. Wazwaz, "Nonlinear variants of the improved Boussinesq equation with compact and noncompact structures," Computers and Mathematics with Applications, vol. 49, no. 4, pp. 565-574, 2005. 


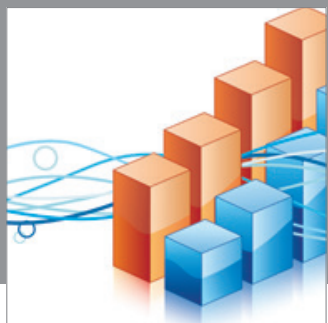

Advances in

Operations Research

mansans

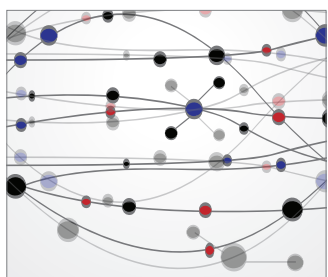

The Scientific World Journal
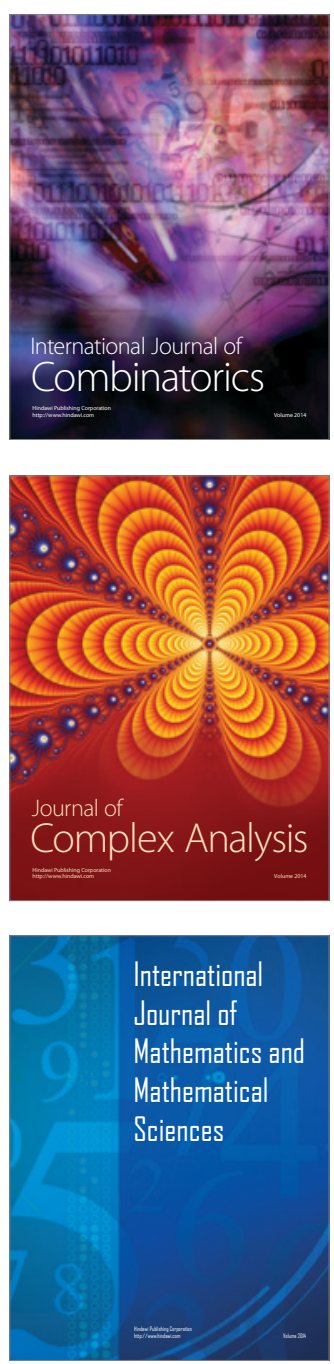
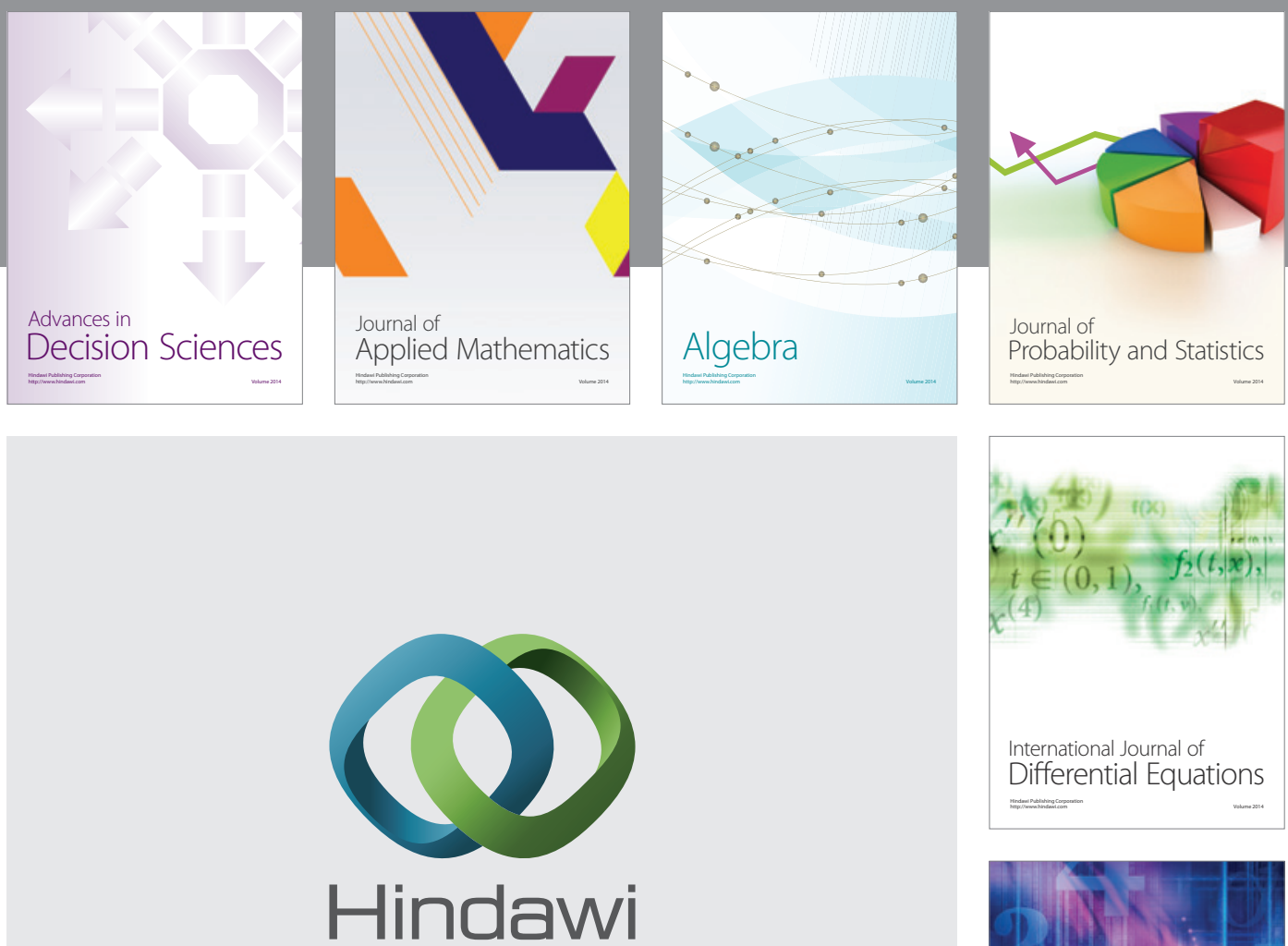

Submit your manuscripts at http://www.hindawi.com
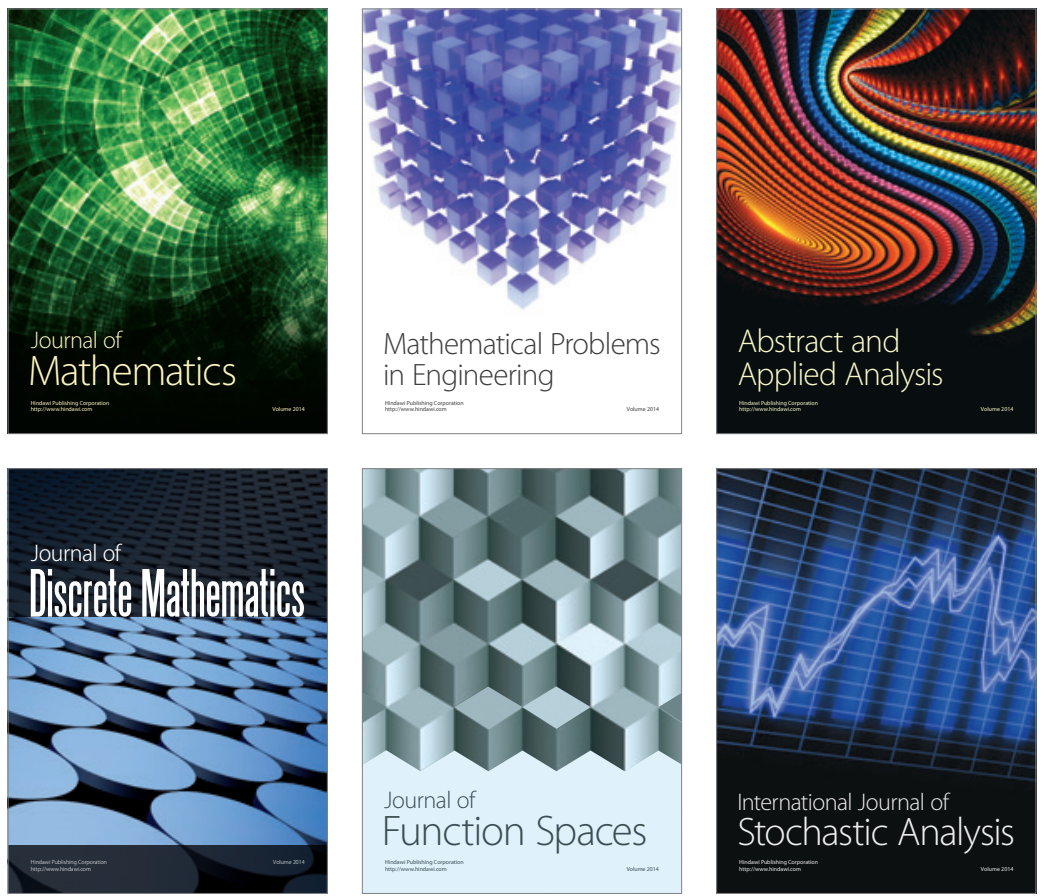

Journal of

Function Spaces

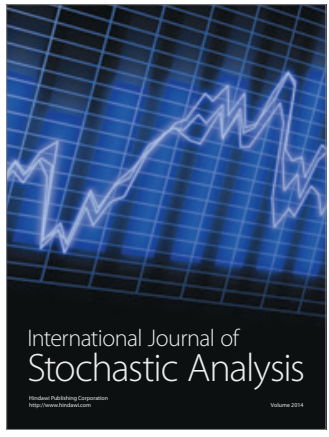

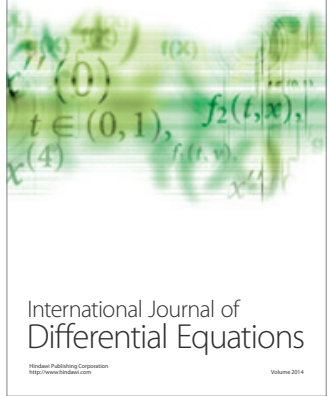
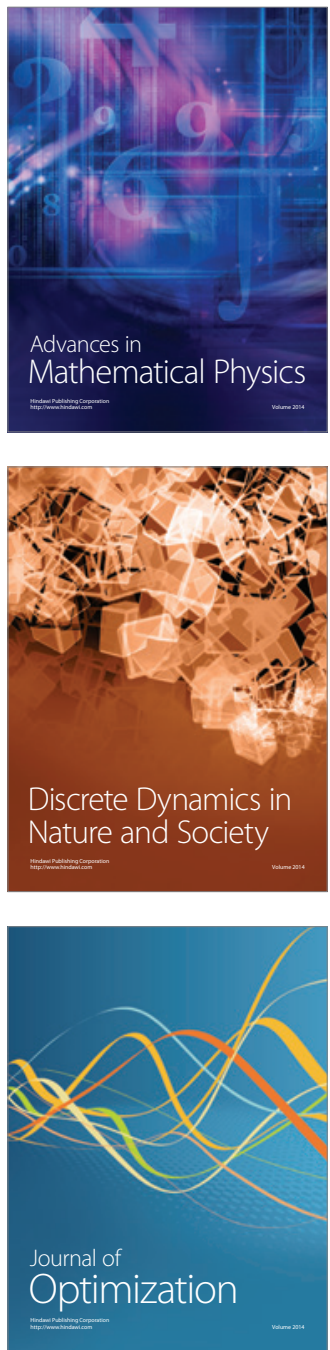